\title{
Search for $\mathrm{E}_{2 g}$ phonon modes in $\mathrm{MgB}_{2}$ single crystals by point-contact spectroscopy
}

\author{
Yu. G. Naidyuk, I. K. Yanson, O. E. Kvitnitskaya \\ B. Verkin Institute for Low Temperature Physics and Engineering, \\ National Academy of Sciences of Ukraine, 47 Lenin Ave., 61103, Kharkiv, Ukraine \\ S. Lee, and S. Tajima \\ Superconductivity Research Laboratory, ISTEC, 1-10-13 Shinonome, Koto-ku, Tokyo 135-0062, Japan
}

(Dated: November 17, 2018)

\begin{abstract}
The electron-phonon interaction in magnesium diboride $\mathrm{MgB}_{2}$ single crystals is investigated by point-contact (PC) spectroscopy. For the first time the electron coupling with $\mathrm{E}_{2 g}$ phonon modes is resolved in the PC spectra. The correlation between intensity of the extremely broad $\mathrm{E}_{2 g}$ modes in the PC spectra and value of the superconducting gap is established. Our observations favor current theoretical models for electron-phonon mediated superconductivity in $\mathrm{MgB}_{2}$ and they better match the harmonic phonons model.
\end{abstract}

PACS numbers: 74.25.Fy, 74.80.Fp, 73.40.Jn

Introduction. During the two year rush in study of superconductivity in $\mathrm{MgB}_{2}$ the electron-phonon coupling has been debated as a main source of the Cooper pairing. Many of theoretical calculations report similar findings: the $\mathrm{E}_{2 g}$ phonon modes, corresponding to the inplane distortions of the boron hexagons, have an enormous coupling to electrons and are responsible for high $\mathrm{T}_{c}$ [1, 2, 3, 4, 5, 6, 7, 8]. Correspondingly, the main feature of the calculated electron-phonon interaction (EPI) function $\alpha^{2} F(\omega)$ is a dominant maximum at the energy

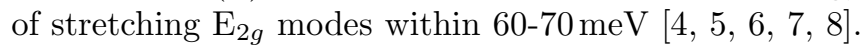
However, in spite of the great progress in computation of $\alpha^{2} F(\omega)$, which is calculated even for various parts of the Fermi surface [7, 8], direct experimental verification for the $\alpha^{2} F(\omega)$ behavior is still lacking.

The importance of the $\mathrm{E}_{2 g}$ modes is evidenced by the Raman spectroscopy studies, which demonstrate strongly damped and broad optical modes with the $\mathrm{E}_{2 g}$ symmetry [5, 9, 10, 11]. Additionally, an anomalous large broadening of the $\mathrm{E}_{2 g}$ phonon modes along the $\Gamma$-A direction is recently observed in single crystals by inelastic X-ray scattering $[12$. It is shown that the dominant contribution to the linewidth is due to a strong electron-phonon interaction (EPI). But both the foregoing experiments deal with phonons and not directly with the $\alpha^{2} F(\omega)$ function.

The two well known fundamental experimental methods for determining $\alpha^{2} F(\omega)$ are point-contact (PC) and tunneling spectroscopy. Both of them were successfully utilized for observation and study of superconducting gap(s) (see, e. g., Refs. 113, 14, 15, 16, 17, 18, 19]). Nevertheless, only a few papers report the search for the phonon structure and EPI in PC [20, 21] or in tunneling spectra 22.

Bobrov et al. [20] communicated reproducible maxima, including a maximum around $60 \mathrm{mV}$, in the $\mathrm{PC}$ spectra for different contacts prepared on the c-axis oriented $\mathrm{MgB}_{2}$ films. In the forthcoming paper Yanson et al. 21] stressed that in the superconducting state the phonon structure observed in the PC spectra was due to the energy dependence of the superconducting order parameter [23, 24]. For strong coupled superconductors this, so called, elastic term 24] exceeds the inelastic EPI contribution to the PC spectra determined directly by $\alpha^{2} F(\omega)$ 25]. Consequently, to separate or to distinguish the elastic and inelastic effects measurements of $\mathrm{PC}$ spectra of $\mathrm{MgB}_{2}$ in the normal state are required. However, the resolution in the point-contact spectroscopy (PCS) is limited by temperature and at $T \geq T_{c} \simeq 40 \mathrm{~K}$ it is above $20 \mathrm{mV}$. On the other hand, the $\mathrm{MgB}_{2}$ thin films have a large critical field above $25 \mathrm{~T}$ [26] to drive them in the normal state at helium temperature.

This paper reports the investigation of $\mathrm{MgB}_{2}$ single crystals by PCS. The advantages of the single crystal as compared to the films in the context of the foregoing discussion are a few times lower critical field, permitting superconductivity to be almost suppress by moderate field along the c-direction [27], the best sample quality (lower residual resistance or larger mean free path) and the possibility of anisotropy study.

The goal of the investigations is to recover the EPI function for $\mathrm{MgB}_{2}$ by means of PCS in order to elucidate the mentioned above issue about role of $\mathrm{E}_{2 g}$ modes in superconductivity of this compound.

Experimental details. The single crystals of $\mathrm{MgB}_{2}$ were grown in a quasi-ternary $\mathrm{Mg}-\mathrm{MgB}_{2}$ - $\mathrm{BN}$ system at a pressure of $4-6 \mathrm{GPa}$ and temperature $1400-1700^{\circ} \mathrm{C}$ for 5 to 60 min. In optimal conditions shiny yellow-colored single crystals with the large side of $0.2-0.7 \mathrm{~mm}$ were yielded. The details of the sample preparation are given elsewhere 27. The estimated resistivity at $40 \mathrm{~K}$ is about $1 \mu \Omega \mathrm{cm}$ and the residual resistivity ratio $R R R=5 \pm 0.1$. A sharp superconducting transition in resistivity is around 38.1$38.3 \mathrm{~K}$ with $\delta T_{c}(10-90) \%=0.2-0.3 \mathrm{~K}$.

The PC's were established in situ at low temperatures by touching of a $\mathrm{Cu}$ or Au electrode by tiny piece (scrap) 
of the $\mathrm{MgB}_{2}$ single crystal. That is because of the small size and the irregular shape the $\mathrm{MgB}_{2}$ crystal was used as a "needle". We always try to align the c axis of the $\mathrm{MgB}_{2}$ single crystal with an applied magnetic field and the contact axis was along the largest side, presumably, perpendicular to the c axis in most cases. Due to lack of special micro-mechanics the accuracy of orientation was held by eye. No more control of the sample orientation was possible during the measurements. Therefore, information about the contact axis orientation with respect to the main crystallographic direction was gained from the analysis of the gap features.

Results and discussion. Study of nonlinear conductivity of metallic PC's allows the EPI function $\alpha^{2} F(\omega)$ to be recovered directly by measuring the second derivative of the $I-V$ characteristic (see, e. g., Ref. 25, 28]):

$$
R_{0}^{-1} \frac{\mathrm{d} R}{\mathrm{~d} V}=\left.A R_{0}^{-1 / 2} \alpha_{\mathrm{PC}}^{2}(\epsilon) F(\epsilon)\right|_{\epsilon=\mathrm{e} V},
$$

where $A$ is the constant, $R=\mathrm{d} V / \mathrm{d} I, R_{0}$ is the contact resistance at zero bias, $\alpha_{\mathrm{PC}}^{2} F(\omega)$ is the PC EPI function, which differs from the Eliashberg EPI function by the presence of factor $K=1 / 2(1-\theta / \tan \theta)$, where $\theta$ is the angle between initial and final momenta of scattered electrons [for the transport and Eliashberg EPI functions the corresponding factors are: $K=(1-\cos \theta)$ and $K=1$, respectively]. From Eq.(11) $\alpha_{\mathrm{PC}}^{2}(\epsilon) F(\epsilon)$ can be expressed via the measured rms signal of the first $V_{1} \propto \mathrm{d} V / \mathrm{d} I$ and the second $V_{2} \propto \mathrm{d}^{2} V / \mathrm{d} I^{2}$ harmonics of a small alternating voltage superimposed on the ramped $d c$ voltage $V$ :

$$
\alpha_{P C}^{2}(\epsilon) F(\epsilon)=\frac{2 \sqrt{2}}{A} R_{0}^{1 / 2} \frac{V_{2}}{V_{1}^{2}} .
$$

It should be emphasized that to discard the influence of the superconducting peculiarities we have analyzed the PC spectra measured at a maximal magnetic field (as high as $9 \mathrm{~T}$ ) which is close to the upper critical field in the $\mathrm{MgB}_{2}$ single crystal [27]) and/or above $\mathrm{T}_{c} \leq 40 \mathrm{~K}$. The common features for all the spectra measured at low temperatures are the residual superconducting structure (the sharp maximum near zero bias) and the continuously raising structureless background above $25-30 \mathrm{mV}$. For a number of curves a smoothed maximum (rather a hump) between $40-80 \mathrm{mV}$ was resolved. The examples of the measured dependences with the maximum are shown in Figs. 1-3. First of all we should stress that the structure in the spectra shown above $30 \mathrm{mV}$ is robust with varying the magnetic field in the range $6-9 \mathrm{~T}$, while the superconducting structure below $30 \mathrm{mV}$ depresses drastically by a magnetic field (see Fig. 1). Secondly, above $30 \mathrm{mV}$ the $\mathrm{d}^{2} V / \mathrm{d} I^{2}(V)$ curves are reproducible for both polarities of the applied voltage. This is in support of the phonon nature of the maxima(um) in $\mathrm{d}^{2} V / \mathrm{d} I^{2}(V)$. From the minima position in the $\mathrm{d} V / \mathrm{d} I$ curves in the insets of Figs. 1 and 2 measured at zero field and low temperature

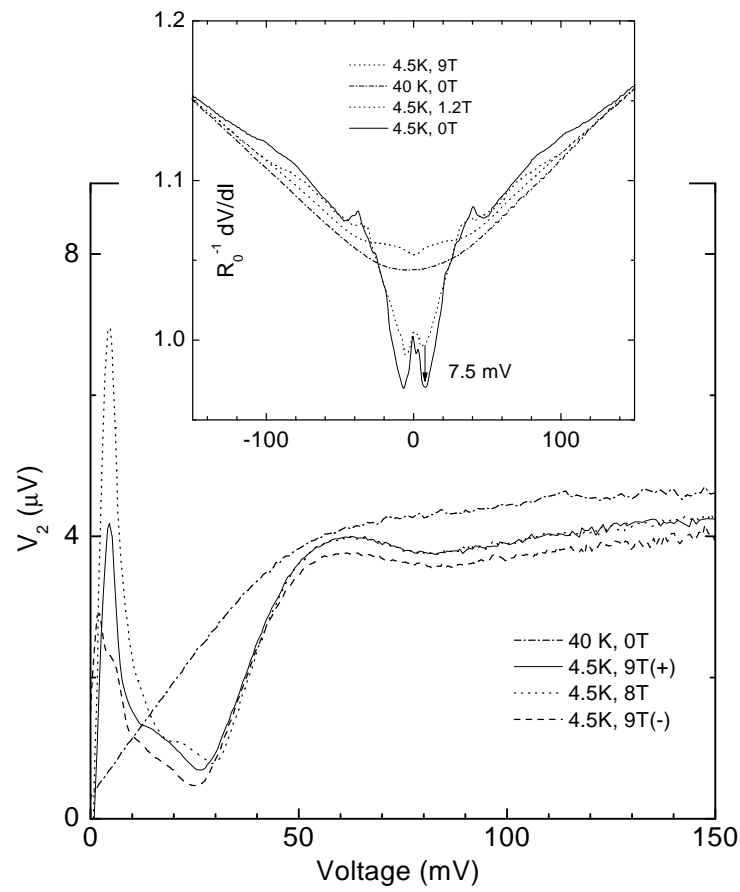

FIG. 1: PC spectra for the $\mathrm{MgB}_{2}-\mathrm{Cu}$ contact $\left(R_{0}=7.2 \Omega\right)$ at $T=4.2 \mathrm{~K}$ in a magnetic field $8 \mathrm{~T}$ (dotted curve) and $9 \mathrm{~T}$ (solid curve). The dashed curve is for the opposite polarity at $9 \mathrm{~T}$. Above $30 \mathrm{mV}$ the curves practically coincide. The dashdotted curve is recorded at zero field at $40 \mathrm{~K}$. The modulation signal $V_{1}(0)$ is $3 \mathrm{mV}$. Inset: the $\mathrm{d} V / \mathrm{d} I(V)$ curves for the same contact. The bottom two curves are for zero and $1.2 \mathrm{~T}$ field and $4.2 \mathrm{~K}$.

we can estimate a gap value which is around $7.5 \mathrm{meV}$ and $2.7 \mathrm{meV}$, respectively. Considering the well fixed by PCS and tunneling fact 16, 19 that a gap of about $7 \mathrm{mV}$ corresponds to the 2-D $\sigma$ bands and a large gap is seen for the direction of the boron planes, we suggest that the spectra in Fig. 1 are measured presumably along the defined direction of the boron sheets. Additional support that we have really observed a large gap in this case is provided by the magnetic field data. A field about $1 \mathrm{~T}$ only slightly modified the gap structure in $\mathrm{d} V / \mathrm{d} I$ (Fig. 1, inset), while according to Ref. 16., this field is sufficient to suppress the small gap. In this connection maxima at about $60 \mathrm{mV}$ in the spectra in Fig. 1 are naturally to connect with the broadened $\mathrm{E}_{2 g}$ phonon modes, which predominate in $\alpha^{2} F(\omega)$ for the $\sigma$ band [7].

Unlike the spectrum in Fig. 1, the spectrum in Fig. 2 with a small gap about $2.7 \mathrm{meV}$ is attributed to the $3-\mathrm{D} \pi$ bands and is governed by the directions out of the boron planes. Correspondingly, the shallow maxima close to 30 and $50 \mathrm{mV}$ reflect bulk (isotropic) phonons, and their correlation with the first two maxima in the phonon DOS [9] or $\alpha^{2} F(\omega)$ [5, 6, 7] is evident. We should also emphasize that the intensity of the spectrum in Fig. 1 is about two times higher than that in Fig. 2, which, according to Eq. (2), results in about 5 times larger value for $\alpha^{2} F(\omega)$ due to the difference in the $\mathrm{PC}$ resistance $R_{0}$. This is 


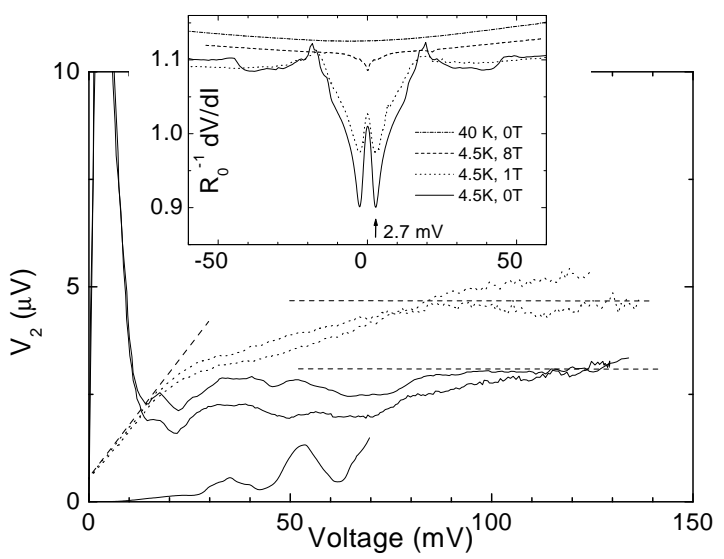

FIG. 2: PC spectra for the $\mathrm{MgB}_{2}-\mathrm{Cu}$ contact $\left(R_{0}=1.5 \Omega\right.$, $V_{1}(0)=3.2 \mathrm{mV}$ ) at $T=4.2 \mathrm{~K}$ and $B=8 \mathrm{~T}$ (solid curves). The dashed curves are recorded at zero field and $40 \mathrm{~K}$. Couple curves are shown for the both polarities of the applied voltage. Dashed straight lines show position of a kink and saturation in the PC spectra. The bottom curve is the low energy part of the isotropic $\alpha^{2} F(\omega)$ [f] smoothed by the experimental resolution about $6 \mathrm{mV}$. Inset: the $\mathrm{d} V / \mathrm{d} I(V)$ curves for the same contact.

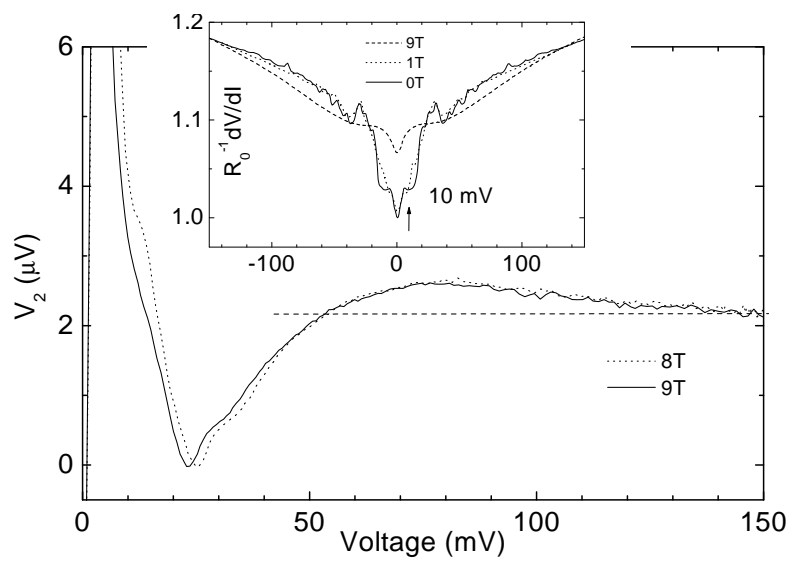

FIG. 3: PC spectra for $\mathrm{MgB}_{2}-\mathrm{Cu}$ contact $\left(R_{0}=7.5 \Omega\right.$, $V_{1}(0)=2.5 \mathrm{mV}$ ) at $T=4.2 \mathrm{~K}$ in a magnetic field $8 \mathrm{~T}$ (dashed curve) and $9 \mathrm{~T}$ (solid curve). Curves are almost the same for the both polarities of the applied voltage. Inset: $\mathrm{d} V / \mathrm{d} I(V)$ curves for the same contact.

also in line with an expected larger strength of EPI in the boron planes.

Take a note of the spectra in Figs. 1 and 2 measured above $T_{c}$ around $40 \mathrm{~K}$. Both of them exhibit a buckle (kink) at the position of the maximum in the corresponding low temperature spectra as well as the saturation above $80 \mathrm{mV}$. This is an additional confirmation of the phonon caused structure in the curves shown.

Fig. 3 depicts an extra example of the PC spectrum with a smooth maximum around $75 \mathrm{mV}$ which also correlates with the dominating maximum in many theoretical $\alpha^{2} F(\omega)$ as well as with the Raman data (see Fig. 4(b)). Note that though $\mathrm{d} V / \mathrm{d} I$ is deviated from the BTK shape because of a minimum at $V=0$ and over-gap maxima,

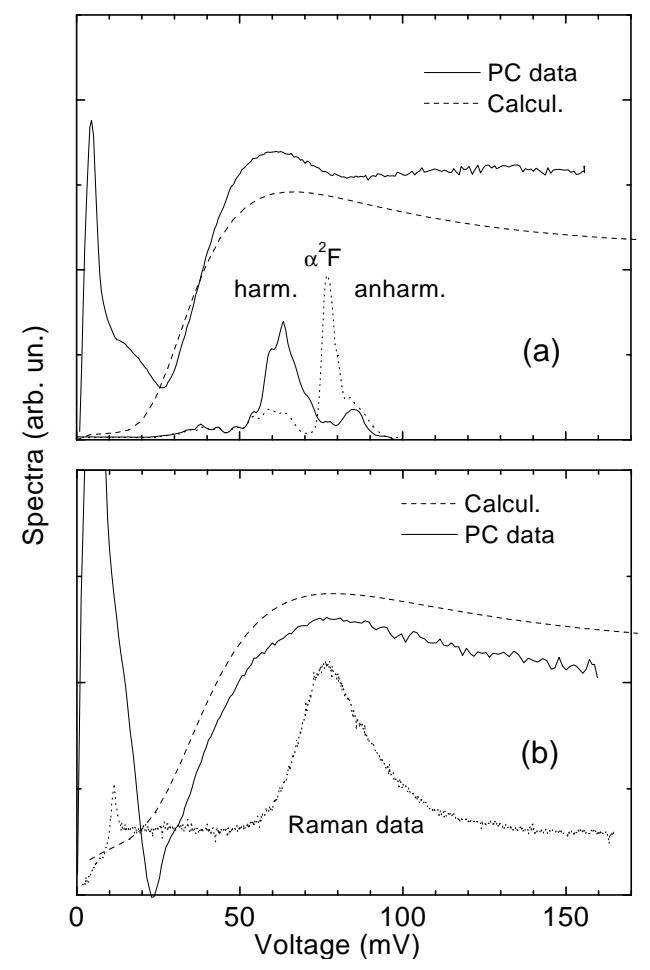

FIG. 4: (a) PC spectrum from Fig.1 transformed into $R_{0}^{-1} \mathrm{~d} R / \mathrm{d} V \propto V_{2} / V_{1}^{2}$ in comparison with the calculated one according to Eq.(3) for the Lorentz-shaped peak at $60 \mathrm{mV}$ and a width of $2 \mathrm{mV}$. The bottom curves show $\alpha^{2} F(\omega) 8$ for harmonic and anharmonic phonons. (b) The PC spectrum from Fig. 3 in comparison with the calculated one according to Eq. (3) for the Lorentz-shaped peak at $70 \mathrm{mV}$ and a width of $15 \mathrm{mV}$. The bottom curve is depolarized Raman spectra of $\mathrm{MgB}_{2}[10]$ at $21 \mathrm{~K}$.

the so-called "gap" minima are observed here at about $|V| \simeq 10 \mathrm{mV}$. Therefore, we attribute these spectra to the $\sigma$ band.

Further comparison of our data with the Raman spectrum shows that the Raman maximum (Fig. 4(b)) is narrower and shifted to a higher energy with respect to that in the PC spectrum in Fig. 4(a). Note that the Raman spectroscopy studies are restricted to the Brillouin zone center, and the Raman maxima, in general, should not correspond, e. g., to the maxima in the phonon DOS or especially in $\alpha_{\mathrm{PC}}^{2} F(\omega)$, which reflects phonons with a large (maximal) momentum.

According to the inelastic X-ray scattering data 12] an anomalous broadening of the $\mathrm{E}_{2 g}$ modes is found to occur along the $\Gamma$-A direction with a width $\delta E=10$ $17 \mathrm{meV}$ and phonon dispersion between 60 and $70 \mathrm{meV}$. This energy range hits the maximum in the spectra in Fig. 1, however, it is fairly broad. Using the width of the $\mathrm{E}_{2 g}$ modes along the $\Gamma$-A direction we estimate the phonon lifetime as $\tau \propto \hbar / \delta E \simeq(4-7) \cdot 10^{-14}$ s. Taking into account the upper value of the sound velocity $s \leq$ $10^{4} \mathrm{~m} / \mathrm{s}$ [30] this leads to the phonon mean free path of the order of the lattice constant. Therefore, the energized 
electron generated nonequilibrium phonons accumulate in the contact region, giving rise to (i) a large background signal 25 and (ii) a thermalization of phonons directly in the $\overrightarrow{\mathrm{PC}}$ and a transition to a thermal regime 25. In the latter case the phonon features in the PC spectra are considerably smeared out.

The PC EPI spectrum in the thermal regime supposing that the phonon part of the resistivity is small compared with the residual resistivity is given by Kulik [25]:

$$
R_{0}^{-1} \frac{\mathrm{d} R}{\mathrm{~d} V}(V)=C \int_{0}^{\infty} \frac{\mathrm{d} \omega}{\omega} \alpha^{2} F(\omega) S(e V / \hbar \omega),
$$

where $C$ is the constant and $S(x)$ represents a smeared around $x=1$ step with a shallow maximum at $x=1.09$. We have calculated the PC spectrum by (3) for $\alpha^{2} F(\omega)$ where the $\mathrm{E}_{2 g}$ mode was simulated by the Lorentz-shaped peak. Comparison with the experimental curves is shown in Fig. 4. The results describe fairly well the spectrum of the contact from Fig. 3 and shows that the spectrum in Fig. 1 has a more distinct maximum, as expected in the thermal regime. The position of this maximum corresponds to the main peak in $\alpha^{2} F(\omega)$ calculated for the harmonic phonon model [8]. This correlates with the statement by Shukla et al. [12 according to which the anharmonic contribution to the phonon linewidth is much smaller compare to the electron-phonon one.

Turn to the EPI inelastic contribution in the PC spectrum, which is about $10 \%$ of the total $\mathrm{PC}$ resistance (see Fig. 1, the inset). it is a few times higher than for the $\mathrm{MgB}_{2}$ thin films 20. However, it is of the same order as that for the non-superconducting transition metal diborides 31], where the parameter EPI is rather low $\lambda \lesssim 0.1$. Conventional explanation considers a deviation from the ballistic transport, leading to a decrease in the intensity of the PC spectra [25, 32]. However, due to the strong variation of $\mathrm{EPI}$ in $\mathrm{MgB}_{2}$ on the Fermi surface and the fact that the large EPI is confined to a small volume in the $\mathbf{k}$-space along the $\Gamma$-A direction [8] the PC EPI function $\alpha_{\mathrm{PC}}^{2} F(\omega)$ can be vastly different compared with the thermodynamic $\alpha^{2} F(\omega)$ one. Therefore, to elucidate the details of EPI in $\mathrm{MgB}_{2}$, the calculation of $\alpha_{\mathrm{PC}}^{2} F(\omega)$ with the mentioned $K$-factor accounting for strong anisotropy of EPI on the Fermi surface and the phonon lifetime effects is very desirable.

Conclusion. We have measured anisotropic PC spectra in the single crystal of $\mathrm{MgB}_{2}$. By virtue of the strong EPI and the short phonon lifetime a very likely close-tothermal-regime state develops for the most PC's, which hinders receiving detailed information about the spectral EPI function. However, certain of the PC's display a spectrum, which corresponds to EPI in the $\sigma$ band, and exhibits damped broad maximum just above $60 \mathrm{meV}$ caused by the $\mathrm{E}_{2 g}$ phonon modes. The $\pi$ band spectrum reveals only lower lying phonon modes. Accordingly, for the first time the experimental manifestation of the anisotropic interaction of electrons with $\mathrm{E}_{2 g}$ phonons is presented. Our observation is also in consistent with the harmonic model of EPI in $\mathrm{MgB}_{2}$.

Acknowledgments. The work was supported by the National Academy of Sciences of Ukraine and by the New Energy and Industrial Technology Development Organization (NEDO) in Japan. We are indebted to A. Golubov and $\mathrm{O}$. Dolgov for providing the $\alpha^{2} F(\omega)$ data. The investigations were carried out in part with the use of the equipment donated by the Alexander von Humboldt Foundation (Germany).

[1] J. M. An and W.E. Pickett, Phys. Rev. Lett. 86, 4366 (2001).

[2] T. Yildirim et al., Phys. Rev. Lett, 87, 037001 (2001)

[3] J. Kortus et al., Phys. Rev. Lett. 86, 4656 (2001).

[4] A. Y. Liu et al., Phys. Rev. Lett. 87087005 (2001).

[5] K.-P. Bohnen et al., Phys. Rev. Lett. 86, 5771 (2001).

[6] Y. Kong et al., Phys. Rev. B, 64, 020501(R) (2001).

[7] A. A. Golubov et al., J. Phys.: Cond. Mat., 14, 1353 (2002).

[8] Hyoung Joon Choi et al., Phys. Rev B, 66, 020513(R) (2002).

[9] B. Renker et al., Phys. Rev. Lett. 88, 067001 (2002).

[10] J. W. Quilty, et al Phys. Rev. Lett., 88, 087001 (2002).

[11] J. Hlinka et al., Phys. Rev. B 64, 140503 (2001); A. F. Goncharov et al., Phys. Rev. B 64, 100509 (2001); D. Lampakis et al., cond-mat/0105447.

[12] Abhay Shukla et al., cond-mat/0209064.

[13] P. Szabó et al., Phys. Rev. Lett. 87137005 (2001).

[14] Y. Bugoslavsky et al., Supercond. Sci. Technol. 15, 526 (2002)

[15] Yu. G. Naidyuk et al., JETP Lett., 75, 238 (2002).

[16] R. S. Gonnelli et al., cond-mat/0208060.

[17] H. Schmidt et al., Phys. Rev. Lett. 88, 127002 (2002).

[18] F. Giubileo et al., Europhys. Lett., 58, 764 (2002).

[19] M. Iavarone et al., Phys. Rev. Lett. 89, 187002 (2002).

[20] N. L. Bobrov et al., in New Trends in Superconductivity, Vol.67 of NATO Science Series II: Math. Phys. and Chem., ed. by J. F. Annett and S. Kruchinin, (Kluwer Acad. Publ., 2002), p.225. (also: cond-mat/0110006).

[21] I. K. Yanson et al., cond-mat/0206170.

[22] A. I. D'yachenko et al., cond-mat/0201200.

[23] I. K. Yanson, in Quantum Mesoscopic Phenomena and Mesoscopic Devices in Microelectronic, Vol.559 of NATO Science Series: Ser. C: Math. and Phys. Sci., ed. by I. O. Kulik and R. Ellialtioglu (Kluwer Acad. Publ., 2000), p.61 (also: cond-mat/0008116).

[24] A. N. Omel'yanchuk et al., Sov. J. Low Temp. Phys. 14, 630 (1988).

[25] I. O. Kulik, Sov. J. Low Temp. Phys. 18, 302 (1992).

[26] C. Buzea and T. Yamashita, Supercond. Sci. Technol. 14, R115 (2001).

[27] S. Lee et al., J. Phys. Soc. of Japan, 70, 2255 (2001).

[28] I. K. Yanson, Sov. J. Low Temp. Phys. 9, 343 (1983).

[29] A. Wexler, Proc. Phys. Soc. 89, 927 (1966).

[30] T. V. Ignatova et al., Low Temp. Phys. 28, 190 (2002).

[31] Yu. G. Naidyuk et al., Phys. Rev. B 66, 140301(R) (2002).

[32] I. O. Kulik and I. K. Yanson, Sov. J. Low Temp. Phys. 
4, 596 (1978). 